\title{
Isogenic human pluripotent stem cell disease models reveal ABRA deficiency underlies cTnT mutation-induced familial dilated cardiomyopathy
}

Dear Editor,

Dilated cardiomyopathy (DCM) is a common form of inherited cardiomyopathy. In the past decades, single mutations in various genes encoding sarcomeric, cytoskeletal, and channel proteins etc. have been found to be associated with DCM (Hershberger et al., 2013; McNally and Mestroni, 2017). However, the mechanisms how single mutations in sarcomeric or structural genes lead to the disease remain elusive. An interesting phenomenon often seen in familial cardiomyopathy is that different single mutations on the same gene can cause either DCM or hypertrophic cardiomyopathy (HCM) (Kathiresan and Srivastava, 2012), which exhibit almost opposite disease phenotypes. DCM is characterized by thinned myocardium and septum, ventricular chamber dilation, and systolic dysfunction (Jefferies and Towbin, 2010; McNally and Mestroni, 2017), while HCM exhibits thickened myocardium and septum, reduced ventricular chamber, and diastolic dysfunction (Richard et al., 2003). At the cellular level, HCM cardiomyocytes exhibit concentric hypertrophy characterized by assembly of myofilaments in parallel and widening of the myocytes. In contrast, DCM cardiomyocytes show eccentric hypertrophy, with assembly of the myofilaments in series and myocyte elongation (Kehat and Molkentin, 2010).

The gene TNNT2, which encodes cardiac troponin $T$ (cTnT), is one of such gene showing ramification of cardiomyopathy phenotypes. Deletion of lysine $210(\Delta K 210)$ in one allele of TNNT2 was found to cause familial DCM. Patients carrying this heterozygous mutation exhibit dilated ventricular chamber and systolic dysfunction leading to progressive heart failure with high mortality (Kamisago et al., 2000). In contrast, deletion of glutamic acid $160(\Delta \mathrm{E} 160)$ in one allele of TNNT2 causes HCM (Watkins et al., 1995). Previous studies showed that the $\Delta \mathrm{K} 210$ mutation and $\triangle \mathrm{E} 160$ mutation causes an opposite myofilament $\mathrm{Ca}^{2+}$ sensitivity and force generation (Morimoto et al., 2002), possibly leading to opposite macroscopic disease phenotypes. The contrast cardiomyopathy phenotypes arisen from these two different cTnT mutations provide us a nice model to explore detailed molecular basis triggering DCM disease development.

To investigate the key factors involved in this disease divergence, we generated isogenic human embryonic stem cell ( $h E S C$ ) lines carrying the cTnT- $\Delta K 210$ or cTnT- $\Delta \mathrm{E} 160$ mutation by TALEN-mediated genomic engineering (Fig. S1A and S1B). This eliminated the genetic variations of induced pluripotent stem cells (iPSCs) even from individuals from the same family. Southern blotting using a probe targeting the PGK-Puro cassette confirmed that no non-specific integration within the genome (Fig. S1C-E). Sanger's sequencing analyses of each of these cell lines further confirmed their heterozygous and homozygous nature (Fig. 1A). We next differentiated the heterozygous and homozygous cTnT- $\Delta \mathrm{K} 210$ and $-\Delta \mathrm{E} 160 \mathrm{hESC}$ toward the cardiac lineage. cTnT- $\Delta \mathrm{K} 210$ and $-\Delta \mathrm{E} 160 \mathrm{hESC}$-cardiomyocytes showed abnormal beating activities (Fig. 1B), which suggested a correlation with cardiac arrhythmia. To examine whether $\Delta \mathrm{K} 210$ and $\Delta \mathrm{E} 160$ mutations have an impact on myofilament organization, we further analyzed cardiomyocytes derived from these mutant hESCs by immunostaining the sarcomeric proteins $\mathrm{CTnT}$ and $\alpha$-actinin. Compared to WT hESC-cardiomyocytes, cTnT- $\Delta \mathrm{K} 210$ and $-\Delta \mathrm{E} 160$ cardiomyocytes showed distinct phenotypes in sarcomeric organization (Fig. 1C). A much higher percentage of heterozygous TNNT2 ${ }^{\mathrm{WT} / \Delta \mathrm{K} 210}$ cardiomyocytes showed DCM hallmarks, such as fewer myofibrils, more irregular disrupted sarcomere organization, and punctate cellular distribution of cTnT, which was even more pronounced in homozygous $T N N T 2^{\Delta K 210 / \Delta K 210}$ cardiomyocytes (Fig. 1D). Ultra-structures analyzed by transmission electron microscopy (TEM) showed $\triangle K 210$ cardiomyocytes exhibited relative irregular Z-lines and expanded endoplasmic reticulum (Fig. 1E). In contrast, heterozygous TNNT2 $2^{\mathrm{WT} / \Delta \mathrm{E} 160}$ cardiomyocytes showed relative denser myofibrils with thickened $Z$ lines. Homozygous $T N N T 2^{\triangle E 160 / \Delta E 160}$ cardiomyocytes showed markedly disrupted myofibrils and Z-lines (Figs. 1E and S2). 


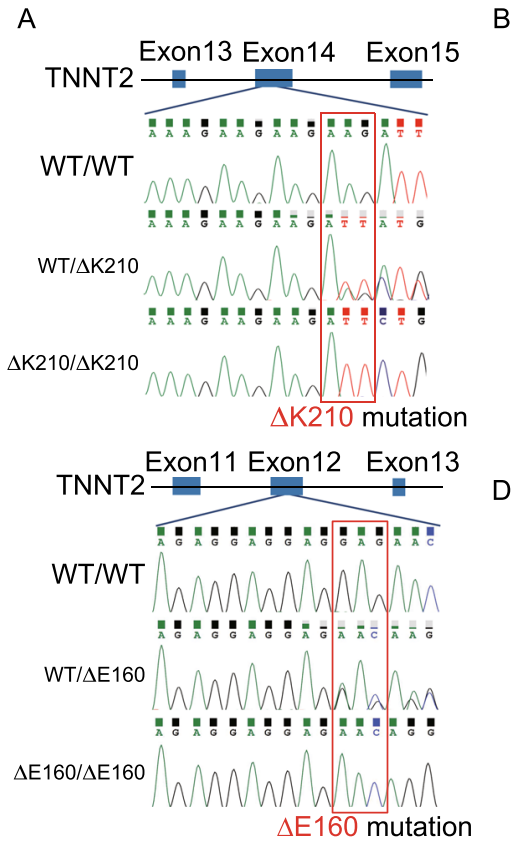

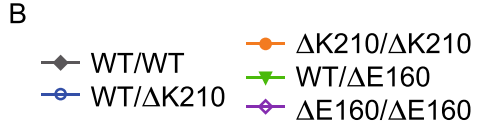
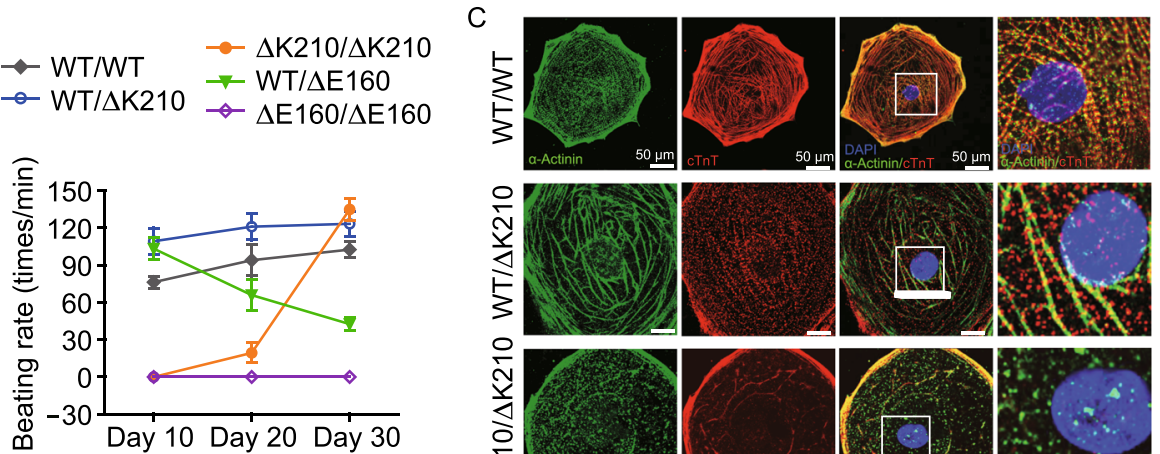

D
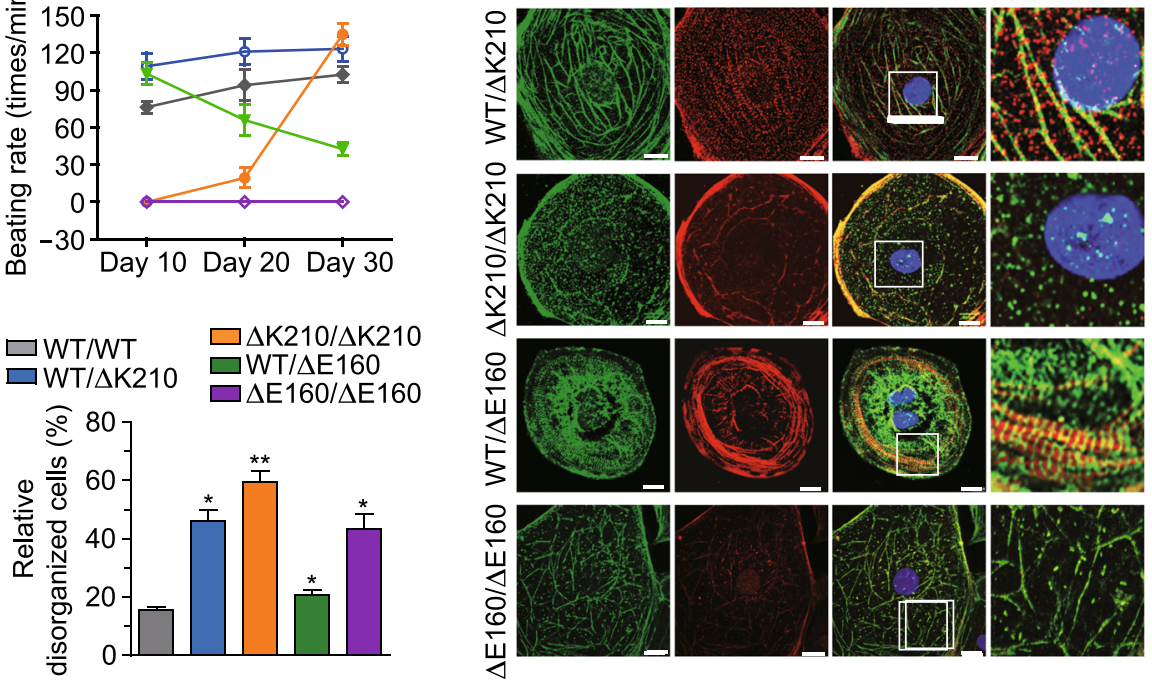

E

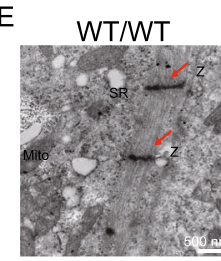
WT/ $\triangle K 210$
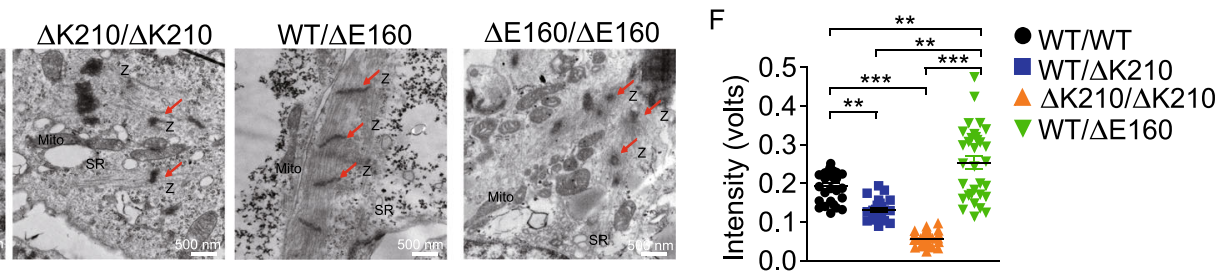

G

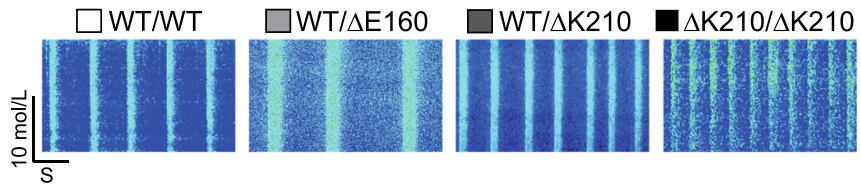

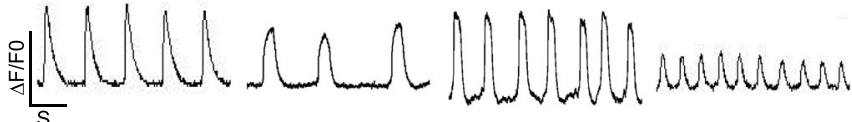

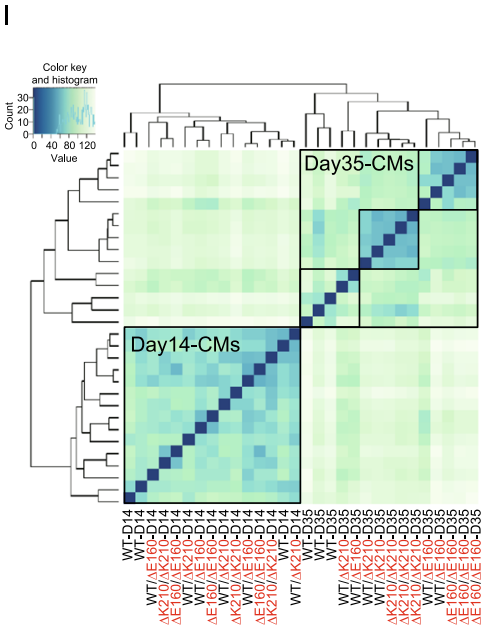

$\mathrm{J}$

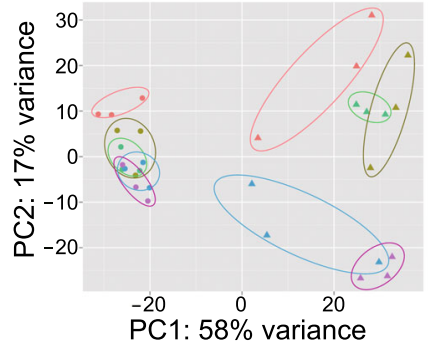

L

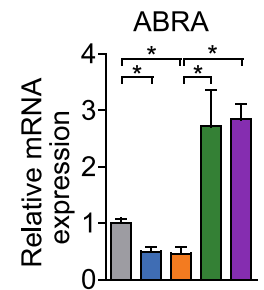

$\mathrm{H}$
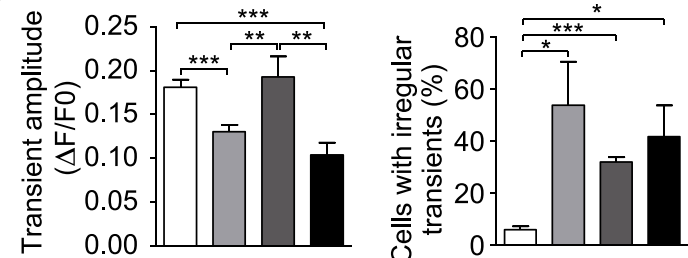

Condition - WT/WT

- WT/ $/ \mathrm{K} 210$

- $\triangle \mathrm{K} 210 / \Delta \mathrm{K} 210$

- WT/AE160

Type

- Day 14
- Day 35

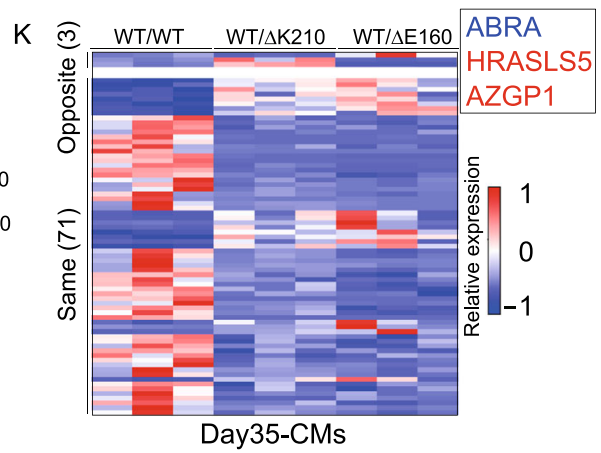

AZGP1

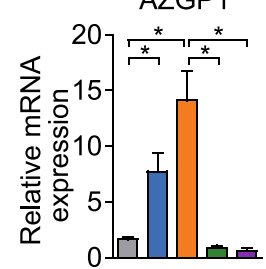

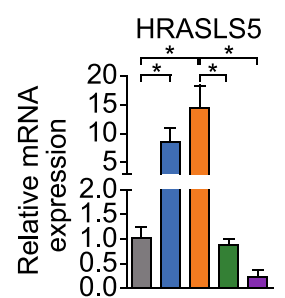

$\square$ WT/WT

$\square \mathrm{WT} / \Delta \mathrm{K} 210$

$\square \Delta \mathrm{K} 210 / \Delta \mathrm{K} 210$

$\square \mathrm{WT} / \Delta \mathrm{E} 160$

- $\Delta \mathrm{E} 160 / \Delta \mathrm{E} 160$ 
4 Figure 1. Generation of $\triangle \mathrm{K} 210$ and $\triangle \mathrm{E} 160$ mutant hESC lines, phenotypic characterizations of mutant cardiomyocytes, and identification of actin binding Rho activating protein (ABRA) as a candidate gene involved in the earliest disease divergence. (A) Sanger's sequencing of PCR-amplified genomic DNA in wildtype (WT), heterozygous, and homozygous $\triangle \mathrm{K} 210$ and $\triangle \mathrm{E} 160 \mathrm{hESCs}$ confirmed deletion of AAG (K210) and GAG (E160) in exon 14 and exon 12, respectively, in the TNNT2 gene. (B) The beating rate (times/min) of hESC-cardiomyocytes of different groups at day 10,20 , and 30 post differentiation. (C) Representative cellular myofilament organization of day 35 single WT, heterozygous and homozygous $\Delta \mathrm{K} 210$ or $\Delta \mathrm{E} 160$ cardiomyocytes immunostained with cTnT (red) and a-actinin (green). Scale bars, $50 \mu \mathrm{m}$. (D) The percentage of cells with disorganized sarcomeric pattern in each group at day 35 post cardiac differentiation. (E) Representative TEM images of myofibrillar organization of day 35 cardiomyocytes in each group. Z, Z-line. Red arrows indicate disorganized or thickened myofibrils. Scale bars, $500 \mathrm{~nm}$. (F) Quantification of the relative spontaneous contraction forces for day 35 single hESC-cardiomyocytes in each group. ( $\mathrm{G}$ and $\mathrm{H}$ ) Representative $\mathrm{Ca}^{2+}$ line scan images of spontaneous $\mathrm{Ca}^{2+}$ transients and statistics of $\mathrm{Ca}^{2+}$ handling parameters of WT, $\Delta \mathrm{K} 210$, and $\Delta \mathrm{E} 160$ cardiomyocytes at day 35 post differentiation. (I and J) Whole transcriptomic RNA-seq profiles and principal component analysis (PCA) showed significant separation between WT, DCM $\triangle \mathrm{K} 210$, and HCM $\triangle \mathrm{E} 160$ cardiomyocytes at day 35 post differentiation. (K) Heatmap of the differentially expressed genes in day $35 \mathrm{WT}, \mathrm{WT} / \Delta \mathrm{K} 210$ and WT/ $\triangle \mathrm{E} 160 \mathrm{hESC}$-cardiomyocytes. Compared with WT, genes showed opposite expression changes are listed in the box. (L) Quantitative PCR verification of $A B R A, A Z G P 1$, and HRASLS5 expression in day $35 \mathrm{WT}, \mathrm{WT} / \Delta \mathrm{K} 210$ and WT/ $\triangle \mathrm{E} 160 \mathrm{hESC}$-cardiomyocytes. ${ }^{*} P<$ $0.05,{ }^{* *} P<0.01$ and ${ }^{* \star *} P<0.001$.

For the cardiomyocyte function, both heterozygous and homozygous cTnT- $\Delta \mathrm{K} 210$ cardiomyocytes exhibited a reduced relative contraction force compared with WT cardiomyocytes, while cTnT- $\Delta \mathrm{E} 160$ cardiomyocytes showed increased contractility (Fig. 1F). Moreover, cTnT- $\Delta$ K210 and $-\Delta \mathrm{E} 160$ cardiomyocytes exhibit a divergent $\mathrm{Ca}^{2+}$ handling properties and more irregular $\mathrm{Ca}^{2+}$ transients at day 35 post differentiation (Fig. $1 \mathrm{G}$ and $1 \mathrm{H}$ ). Overall, these results showed that heterozygous $\Delta \mathrm{K} 210$ and $\Delta \mathrm{E} 160$ cardiomyocytes largely recapitulated cellular phenotypes of DCM and $\mathrm{HCM}$ respectively, while homozygous $\Delta \mathrm{K} 210$ and $\triangle \mathrm{E} 160$ cardiomyocytes showed more severe but abnormal cellular phenotypes.

Since DCM or HCM is progressively developed in patients' lifetime, we hypothesized that genes involved in the earliest disease development and divergence will be valuable targets for interfering the disease. In this study, we used $\Delta \mathrm{E} 160$ cardiomyocytes as a contrast to look for the key genes involved in the earliest disease progress during DCM development. Whole transcriptomic analyses of day 14 and day $35 \Delta K 210$ and $\Delta E 160$ cardiomyocytes was performed using RNA-sequencing. Day 14 and day 35 cardiomyocytes exhibited distinct gene expression patterns and automatically grouped separately into two large clusters (Fig. 1I and $1 \mathrm{~J})$, suggesting they are at different maturation stages. Within the cluster of day 35 cardiomyocytes, it began to show differential gene expression patterns and almost automatically sub-clustered into WT, $\triangle \mathrm{K} 210 \mathrm{DCM}$, and $\triangle \mathrm{E} 160 \mathrm{HCM}$ groups (Fig. 1I and 1J). To further narrow down the candidate genes in early disease development and divergence of $\mathrm{DCM}$ and $\mathrm{HCM}$, we next compared those differentially expressed genes in day $35 \Delta \mathrm{K} 210$ and $\Delta \mathrm{E} 160$ cardiomyocytes (the time that showed disease divergence) and searched for those genes exhibiting an opposite expression. As shown in Fig. 1K, there were 74 overlapped genes and among which only 3 genes showed opposite direction in expression changes. Of the 3 genes, ABRA (actin binding Rho activating protein, also known as striated muscle activator of Rho signaling (STARS)) were downregulated in $T N N T 2^{\mathrm{WT} / \Delta \mathrm{K} 210} \mathrm{DCM}$ cardiomyocytes and upregulated in $T N N T 2^{\mathrm{WT} / \triangle \mathrm{E} 160} \mathrm{HCM}$ cardiomyocytes. The other two genes HRASLS5 and AZGP1, which are strongly linked to metabolism, were up-regulated in $T N N T 2^{\mathrm{WT} / \Delta \mathrm{K} 210}$ cardiomyocytes and down-regulated in TNNT2 ${ }^{\mathrm{WT} / \Delta \mathrm{E} 160}$ cardiomyocytes (Fig. 1K). Expression of $A B R A, A Z G P 1$, and HRASLS5 in day 35 cardiomyocytes were further validated by quantitative PCR (Fig. 1L). Since ABRA was shown upregulated in skeletal muscle hypertrophy and down-regulated in atrophy in human patients (Lamon et al., 2009), which is closely corresponding to heart muscle hypertrophy and dilation, we speculated that ABRA play an important role in the earliest disease divergence.

ABRA is a cardiac and skeletal muscle-specific actinbinding protein, which specifically localizes to the $Z$ disc and M-line and directly binds actin (Arai et al., 2002). Previous studies demonstrated that ABRA activates the Rho signaling and serum response factor (SRF) mediated transcription by allowing nuclear translocation of MRTF-A and -B. ABRA also promotes actin polymerization by increasing the binding of G-actin to F-actin, strengthens myofilaments of striated muscle cells and modulates the heart response to stress signaling, which may function as a cytoskeletal intermediary of stress sensing and intracellular signaling regulation (Arai et al., 2002; Kuwahara et al., 2007; Wallace et al., 2012). Compared to WT cardiomyocytes, ABRA showed a striated localization pattern but tended to localize to the center of $T N N T 2^{\mathrm{WT} / \Delta \mathrm{K} 210}$ cells and more disrupted in TNNT2 ${ }^{\Delta \mathrm{K} 210 /}$ ${ }^{\triangle K} 210$ cells (Fig. S3A). ABRA and CTnT protein levels were also decreased in cTnT- $\Delta \mathrm{K} 210$ cardiomyocytes (Fig. S3B and S3C). Overexpression of ABRA using lentivirus in cTnT$\triangle \mathrm{K} 210$ cardiomyocytes also rescued the DCM-relevant cellular phenotypes, such as sarcomere disorganization, punctate distribution of cTnT and decreased contractile force (Fig. S3D-H). We reasoned that down-regulation of ABRA in cTnT- $\Delta$ K210 cardiomyocytes reduced F-actin filament 
A

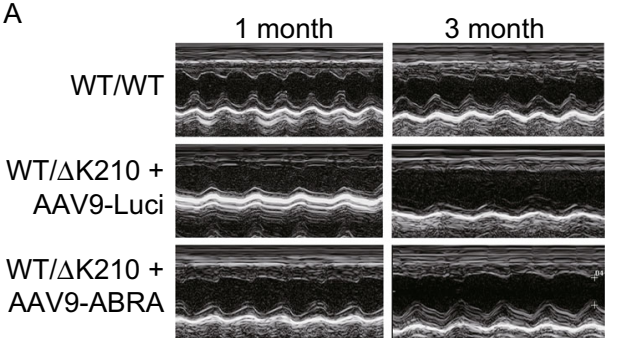

C
D

$\square$ WT

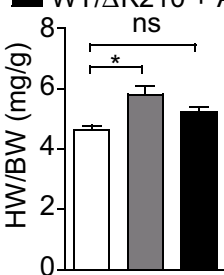

F

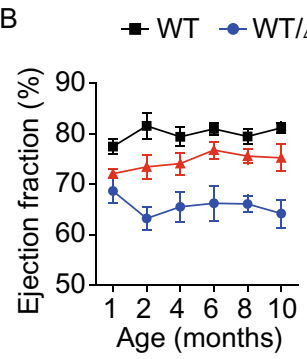

E

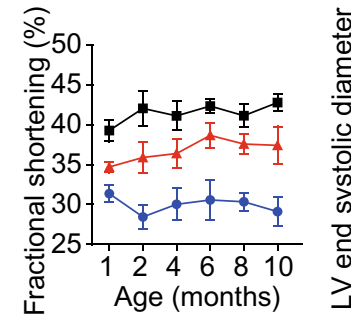

WT/AK210+ AAV9-Luci
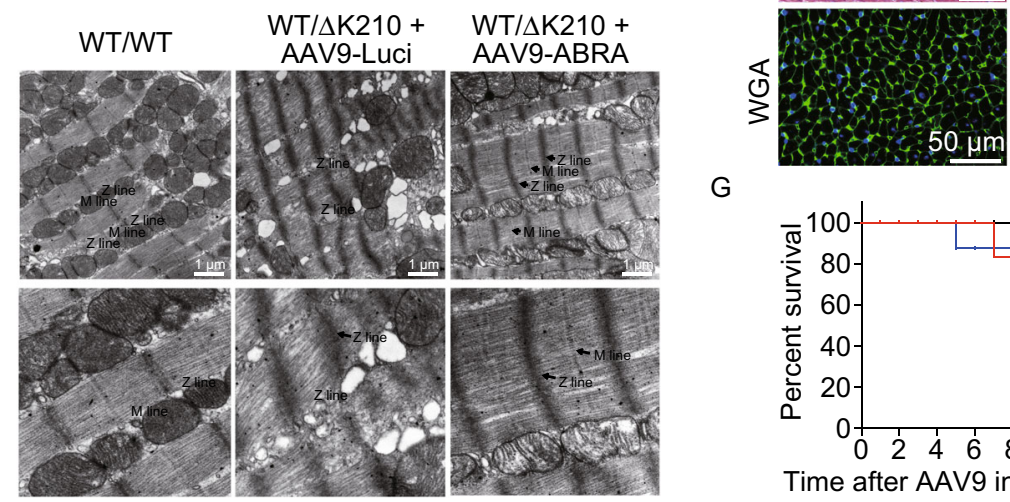

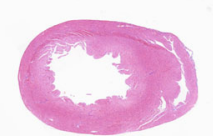

쏘
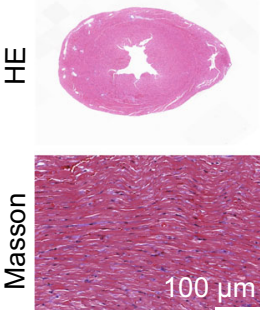

G

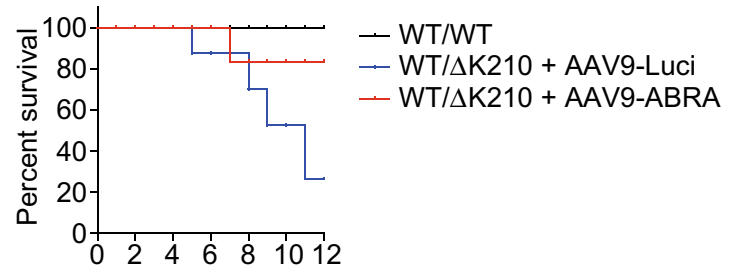

Time after AAV9 injection (months)
$\mathrm{H}$

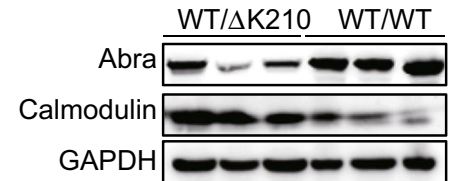

J

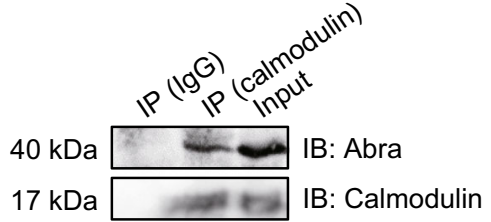

L $\square$ WT/WT

$\square$ WT/ $\Delta K 210+$ AAV9-Luci

- WT/AK210 + AAV9-ABRA

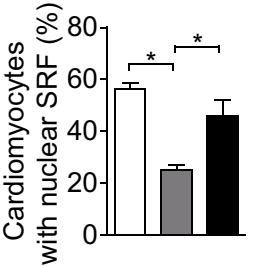

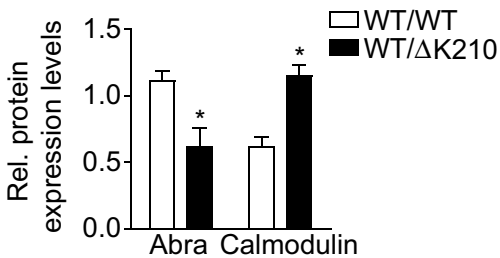

K

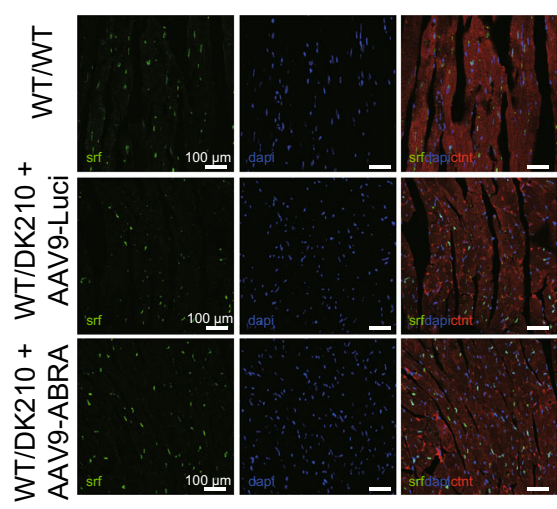

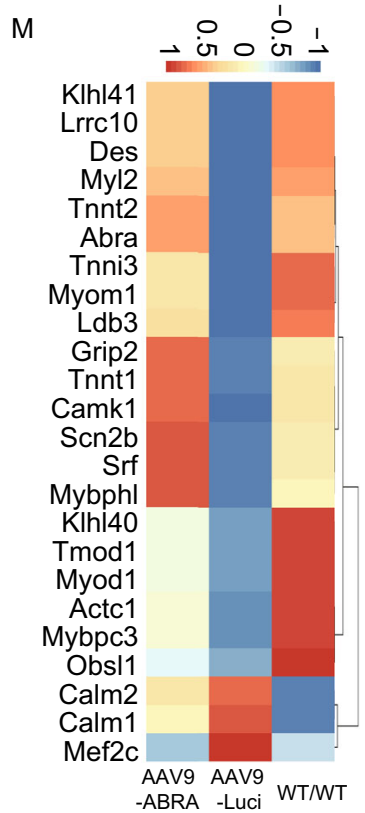


4 Figure 2. Cardiac-specific expression of ABRA rescued DCM phenotypes. (A) Representative M-mode echocardiography recordings of 1-month-old and 3-months-old WT, AAV9Luci injected cTnT- $\Delta K 210$, and AAV9-ABRA injected cTnT$\triangle K 210$ mice. (B) Serial echocardiographic measurements of ejection fraction (EF), fractional shortening (FS), left ventricular end systolic internal diameter (LVIDs) at different time points post virus injection. (C and D) Overall heart morphology and heart weight /body weight (HW/BW) ratio of the whole hearts 3 months after AAV9 virus injection. ( $E$ and $F$ ) Representative $\mathrm{H} \& E$, Masson's trichrome, and WGA staining (E), was well as TEM images $(F)$ of heart sections 3 months after AAV9 virus injection. Scale bars: $100 \mu \mathrm{m}$ (Masson), $50 \mu \mathrm{m}$ (WGA), $1 \mu \mathrm{m}$ (TEM). (G) survival curves for WT, AAV9-Luci injected cTnT$\triangle \mathrm{K} 210$, and AAV9-ABRA injected cTnT- $\Delta \mathrm{K} 210$ mice. (H and I) Western blot assessment and quantification of calmodulin and Abra protein levels in heart tissues of 3-months-old WT and cTnT- $\Delta$ K210 mice. A pan-calmodulin antibody was used. (J) Co-immunoprecipitation of calmodulin and Abra from heart tissue extraction of WT mice. (K) Immunofluorescence staining of SRF in mice heart tissues of different groups 3 months after AAV9 virus injection. Scale bars, $100 \mu \mathrm{m}$. (L) Quantification of percentage of cardiomyocytes exhibiting positive nuclear SRF staining. (M) RNA-seq heatmap profiling of SRF-regulated muscle genes 3 months after AA9 virus injection. ${ }^{*} P<0.05$. ${ }^{\star \star} P<0.01$..

formation and may directly weaken myofilament structures, thereby leading to decreased cell contractility and DCM-relevant phenotypes.

We next investigated whether heart-specific expression of ABRA could reverse DCM phenotypes of the cTnT- $\triangle \mathrm{K} 210$ $\mathrm{DCM}$ mice in vivo. The results showed reduced ABRA protein in cTnT- $\Delta$ K210 mice (Fig. S4A), staining the Z-lines with a-actinin and A-bands with tropmodulin (TMOD1) in cTnT$\Delta K 210$ mice further indicated the Z-lines were relative intact but A-bands were disrupted (Fig. S4B). Recombinant adenoassociated virus 9 (AAV9) with cardiac-specific cTnT promoter driving ABRA expression (AAV9-ABRA) was constructed and AAV9 with cTnT promoter driving luciferase (AAV9-Luci) served as a control. AAVs were injected at 2-3 days after birth through intraperitoneal injection. The luciferase protein was detected only in the hearts, which validated the virus performance (Fig. S5A).

Mice heart function was measured every month after AAV injection by echocardiography. It showed that impaired heart functions were effectively rescued in AAV9-ABRA treated cTnT- $\Delta$ K210 mice at 3 months (Fig. 2A). Left ventricular ejection fraction (EF) and fractional shortening (FS) were reversed to a level close to WT values (Fig. 2B). The left ventricular end-systolic internal diameter (LVIDs) and LV posterior wall (LVPW) thickness of cTnT- $\Delta \mathrm{K} 210$ mice treated with AAV9-ABRA were significantly improved (Figs. $2 B$ and $S 5 B)$. Heart size and heart weight/body weight value of AAV9-ABRA treated cTnT- $\triangle \mathrm{K} 210$ mice were also much smaller and close to those of the WT (Fig. 2C and 2D). Histological analyses showed that AAV9-Luci treated cTnT$\triangle K 210$ mice developed cardiac dilation with enlarged cardiomyocytes and increased myocardial fibrosis, while AAV9ABRA treated mice reversed these typical DCM phenotypes and pathological cardiac remodeling (Fig. 2E). Moreover, AAV9-ABRA treatment restored the disrupted sarcomeric $A$-bands and $M$ lines in cTnT- $\triangle K 210$ mice, although the sarcomere length still seemed compact (Fig. 2F). AAV9ABRA treatment also improved overall survival rate of cTnT$\triangle K 210 \mathrm{DCM}$ mice (Fig. 2G). These results indicated that the DCM phenotypes in cTnT- $\triangle \mathrm{K} 210$ mice were markedly reversed by cardiac-specific ABRA expression. We next used the classical cTnT-R141W knock-in DCM mice (Juan et al., 2008) to examine whether ABRA heart-specific expression is effective in other cTnT mutation-induced familial DCM. As shown in Fig. S6, impaired heart functions in cTnT-R141W mice were rescued after AAV9-ABRA injection.

In addition, our $\mathrm{Ca}^{2+}$ imaging results indicated that the cTnT- $\Delta$ K210 mutation induced abnormal $\mathrm{Ca}^{2+}$ handling (Fig. 1G) and significantly upregulated calmodulin in the heart of cTnT- $\Delta$ K210 mice (Fig. $2 \mathrm{H}$ and 2l). Previous study showed that the $\mathrm{Ca}^{2+}$-dependent protein calmodulin directly binds to the $\mathrm{N}$-terminus of $\mathrm{ABRA}$ and negatively regulates muscle gene expression induced by the ABRA-serum response factor (SRF) pathway (Furuya et al., 2016), thereby linking $\mathrm{Ca}^{2+}$-signaling to ABRA-mediated gene expression. Immunoprecipitation assays showed that calmodulin binds to ABRA in cardiomyocytes (Fig. 2J). We then examined whether ABRA mediated SRF activity was changed in cTnT$\triangle \mathrm{K} 210$ mice. Compared to WT mice, nucleus translocation of SRF was significantly reduced in heart tissues of cTnT$\Delta \mathrm{K} 210$ mice (Fig. $2 \mathrm{~K}$ and $2 \mathrm{~L}$ ). SRF-regulated muscle and contractile genes were significantly downregulated in cTnT$\triangle K 210$ mice and increased after AAV9-ABRA treatment as shown by whole transcriptome RNA-sequencing and further confirmed by QPCR (Figs. 2M and S7). These results suggest that the cTnT- $\Delta \mathrm{K} 210$ mutation causes irregular $\mathrm{Ca}^{2+}$ handling in myofilaments and a resulted up-regulation of calmodulin, which negatively regulates ABRA-SRF activity in cardiomyocytes and compromised downstream muscle related gene expression.

Our current study showed that ABRA deficiency and compromised downstream SRF-regulated muscle gene expression play a role in cTnT mutation induced-familial DCM. ABRA could be a therapeutic gene for DCM patients carrying cTnT mutations. Our findings in this study may also serve as a new strategy in discovering early disease-associated genes for other mutation-caused familial DCM. 


\section{FOOTNOTES}

This work was supported by the National Natural Science Foundation of China (NSFC No.82070391, N.S.), the Postdoctoral Science Foundation (No.KLH1322109, B.L.), the Young Elite Scientist Sponsorship Program by CAST (2018QNRC001), the Haiju program of National Children's Medical Center EK1125180102, and the National Key R\&D Program of China 2018 YFC2000202 (N.S.).

We thank Dr. Lianfeng Zhang at Peking Union Medical College for his kindly provision of the cTnT-R14W mice. We apologize to people whose work was relevant to but not cited in this study due to limited space.

Bin Li, Yongkun Zhan, Qianqian Liang, Chen Xu, Xinyan Zhou, Huanhuan Cai, Yufan Zheng, Yifan Guo, Lei Wang, Wenqing Qiu, Baiping Cui, Chao Lu, Ruizhe Qian, Ping Zhou, Haiyan Chen, Yun Liu, Sifeng Chen, Xiaobo Li, Ning Sun declare that there is no conflict of interests.

All institutional and national guidelines for the care and use of laboratory animals were followed.

The data, analytic methods, and study materials will be made available to other researchers for purposes of reproducing the results or replicating the procedure. The RNA-seq data have been deposited in GEO (Gene Expression Omnibus) with accession code GSE154096 and GSE154097.

B.L., Y.K.Z., and Q.Q.L performed and interpreted the experiments and wrote the manuscript. X.Y.Z. and H.H.C. performed H\&E staining and MEA recording. C.X, Y.F.Z., Y.F.G, L.W., and W.Q.Q performed in vitro experiments. X.B.L., C.L., R.Z.Q and P.Z provided experimental advice. H.Y.C. performed echocardiography. Y.L. performed RNA sequencing and bioinformatics analyses. X.W. provided experimental advice. N.S. conceived the idea and experiments, provided experimental assistance, manuscript writing and funding support.

Bin $\mathrm{Li}^{1,2}$, Yongkun Zhan ${ }^{1}$, Qianqian Liang ${ }^{1,4}$, Chen $X \mathrm{u}^{1,4}$, Xinyan Zhou ${ }^{1}$, Huanhuan Cai ${ }^{1}$, Yufan Zheng ${ }^{1}$, Yifan Guo ${ }^{1}$, Lei Wang ${ }^{6}$, Wenqing Qiu ${ }^{6}$, Baiping Cui ${ }^{1}$, Chao $\mathrm{Lu}^{1,4}$, Ruizhe Qian ${ }^{1,4}$, Ping Zhou ${ }^{1}$, Haiyan Chen ${ }^{5}$, Yun Liu ${ }^{6}$, Sifeng Chen ${ }^{1}$, Xiaobo $\mathrm{Li}^{1}$, Ning Sun ${ }^{1,3,4 \bowtie}$

${ }^{1}$ Department of Physiology and Pathophysiology, State Key Laboratory of Medical Neurobiology, School of Basic Medical Sciences, Fudan University, Shanghai 200032, China

2 Shanghai Institute of Precision Medicine, Ninth People's Hospital, Shanghai Jiao Tong University School of Medicine, Shanghai 200025, China

3 Shanghai Key Lab of Birth Defect, Children's Hospital of Fudan University, Shanghai 201102, China

${ }^{4}$ Shanghai Key Laboratory of Clinical Geriatric Medicine, Research Center on Aging and Medicine, Fudan University, Shanghai 200032, China

${ }^{5}$ Department of Echocardiography, Zhongshan Hospital, Fudan University, Shanghai 200032, China

${ }^{6}$ Department of Biochemistry, School of Basic Medical Sciences, Fudan University, Shanghai 200032, China

$\triangle$ Correspondence: sunning@fudan.edu.cn (N. Sun)

Accepted March 8, 2021

\section{OPEN ACCESS}

This article is licensed under a Creative Commons Attribution 4.0 International License, which permits use, sharing, adaptation, distribution and reproduction in any medium or format, as long as you give appropriate credit to the original author(s) and the source, provide a link to the Creative Commons licence, and indicate if changes were made. The images or other third party material in this article are included in the article's Creative Commons licence, unless indicated otherwise in a credit line to the material. If material is not included in the article's Creative Commons licence and your intended use is not permitted by statutory regulation or exceeds the permitted use, you will need to obtain permission directly from the copyright holder. To view a copy of this licence, visit http:// creativecommons.org/licenses/by/4.0/.

\section{REFERENCES}

Arai A, Spencer JA, Olson EN (2002) STARS, a striated muscle activator of Rho signaling and serum response factor-dependent transcription. J Biol Chem 277:24453-24459

Furuya Y, Denda M, Sakane K, Ogusu T, Takahashi S, Magari M, Kanayama N, Morishita R, Tokumitsu H (2016) Identification of striated muscle activator of Rho signaling (STARS) as a novel calmodulin target by a newly developed genome-wide screen. Cell Calcium 60:32-40

Hershberger RE, Hedges DJ, Morales A (2013) Dilated cardiomyopathy: the complexity of a diverse genetic architecture. Nat Rev Cardiol 10:531-547

Jefferies JL, Towbin JA (2010) Dilated cardiomyopathy. The Lancet 375:752-762

Juan F, Wei D, Xiongzhi Q, Ran D, Chunmei M, Lan H, Chuan Q, Lianfeng $Z$ (2008) The changes of the cardiac structure and function in cTnTR141W transgenic mice. Int J Cardiol 128:83-90

Kamisago M, Sharma SD, DePalma SR, Solomon S, Sharma P, McDonough B, Smoot L, Mullen MP, Woolf PK, Wigle ED et al (2000) Mutations in sarcomere protein genes as a cause of dilated cardiomyopathy. N Engl J Med 343:1688-1696

Kathiresan S, Srivastava D (2012) Genetics of human cardiovascular disease. Cell 148:1242-1257

Kehat I, Molkentin JD (2010) Molecular pathways underlying cardiac remodeling during pathophysiological stimulation. Circulation 122:2727-2735

Kuwahara K, Teg Pipes GC, McAnally J, Richardson JA, Hill JA, Bassel-Duby R, Olson EN (2007) Modulation of adverse cardiac remodeling by STARS, a mediator of MEF2 signaling and SRF activity. J Clin Investig 117:1324-1334

Lamon S, Wallace MA, Leger B, Russell AP (2009) Regulation of STARS and its downstream targets suggest a novel pathway involved in human skeletal muscle hypertrophy and atrophy. J Physiol 587:1795-1803

McNally EM, Mestroni L (2017) Dilated cardiomyopathy: genetic determinants and mechanisms. Circ Res 121:731-748

Morimoto S, Lu QW, Harada K, Takahashi-Yanaga F, Minakami R, Ohta M, Sasaguri T, Ohtsuki I (2002) Ca(2+)-desensitizing effect of a deletion mutation Delta K210 in cardiac troponin $\mathrm{T}$ that 
causes familial dilated cardiomyopathy. Proc Natl Acad Sci USA 99:913-918

Richard P, Charron P, Carrier L, Ledeuil C, Cheav T, Pichereau C, Benaiche A, Isnard R, Dubourg O, Burban M et al (2003) Hypertrophic cardiomyopathy: distribution of disease genes, spectrum of mutations, and implications for a molecular diagnosis strategy. Circulation 107:2227-2232

Wallace MA, Lamon S, Russell AP (2012) The regulation and function of the striated muscle activator of rho signaling (STARS) protein. Front Physiol 3:469
Watkins $H$, McKenna WJ, Thierfelder L, Suk HJ, Anan R, O'Donoghue A, Spirito P, Matsumori A, Moravec CS, Seidman JG et al (1995) Mutations in the genes for cardiac troponin T and alpha-tropomyosin in hypertrophic cardiomyopathy. N Engl J Med 332:1058-1064

Bin Li, Yongkun Zhan, and Qianqian Liang have contributed equally to this work.

Electronic supplementary material The online version of this article (https://doi.org/10.1007/s13238-021-00843-w) contains supplementary material, which is available to authorized users. 